\title{
RELATIVISTIC SELFINTERACTING PARTICLE-ANTIPARTICLE SYSTEM OF BOSONS
}

\author{
D. Anchishkin ${ }^{1 *}$, V. Gnatovskyy ${ }^{2}$, D. Zhuravel ${ }^{1}$, V. Karpenko ${ }^{2}$ \\ ${ }^{1}$ Bogolyubov Institute for Theoretical Physics, National Academy of Sciences of Ukraine, 03143 Kyiv, \\ Ukraine \\ ${ }^{2}$ Taras Shevchenko National University of Kyiv, 03022 Kyiv, Ukraine \\ *e-mail:dmanch@bitp.kiev.ua
}

\begin{abstract}
Thermodynamic properties of a system of an interacting boson particles and antiparticles at high temperatures are studied within the framework of the thermodynamically consistent Skyrme-like mean-field model. The mean field contains both attractive and repulsive terms. Self-consistency relations between the mean field and thermodynamic functions are derived. We assume a conservation of the isospin density for all temperatures. It is shown that, independently of the strength of the attractive mean field, at the critical temperature $T_{\mathrm{c}}$ the system undergoes the phase transition of second order to the Bose-Einstein condensate, which exists in the temperature interval $0 \leq T \leq T_{\mathrm{c}}$. It is obtained that the condensation represents a discontinuity of the derivative of the specific heat at $T=T_{\mathrm{c}}$.

Keywords: boson system, particles and antiparticles, Bose-Einstein condensation.
\end{abstract}

Received 12.10.2020; Received in revised form 15.11.2020; Accepted 01.12.2020

\section{Introduction}

The phase structure of hot and dense hadronic matter have attracted considerable interest in recent years and is a subject of active research $[1,2]$. In these regimes the QCD motivated effective models and lattice simulations indicate that the chiral symmetry restoration and the deconfinement phase transition should take place at high temperatures. In particular, the knowledge of the phase structure of the meson systems, in the regime of finite temperatures and isotope spin (isospin) densities is crucial for understanding of a wide range of phenomena from nucleusnucleus collisions to neutron stars and cosmology. Meanwhile, studying the meson systems has its own specifics: it is necessary to account for a possibility of the Bose-Einstein condensation of interacting bosonic particles. Investigations of the properties of a dense and hot pion gas is quite strongly inspired by the experiments at RHIC and LHC $[3,4]$, where at midrapidity it was discovered a formation of the medium with low baryon numbers.

The problem of the Bose-Einstein condensation of pi-mesons (K-mesons etc.) has been studied previously, starting from the pioneer works of A.B. Migdal and coworkers [5-8] and later by many authors using different models and methods. A possible formation of classical pion fields in relativistic nucleus-nucleus collisions was discussed in refs. [9-12]. In more recent studies [13-17], pionic systems with a finite isospin chemical potential and low temperatures have been considered. First-principles lattice calculations provide a solid basis for our knowledge of the finite temperature regime. New results concerning dense pionic systems have been obtained recently using lattice methods $[18,19]$.

In the present paper we consider the system of bosons, which we name just conventionally as "pions". This preference is made because the charged $\pi$-mesons are the lightest hadrons that couple to the isospin chemical potential. On the other hand, the pions are the lightest nuclear boson particles and thus, an account for "temperature creation" of particle-antiparticle pairs is a relevant problem on the basis of quantum-statistical approach. As a matter of fact, an effective dynamics of the charged pions is described by chiral perturbation theory [13]. However, we investigate the self-interacting many-boson system in the framework of thermodynamic meanfield approach and we consider the present studies as a toy-model which can help us to understand the processes of the Bose-Einstein condensation and phase transitions in the wide range of temperatures and densities. 
The investigation presented is, in some sense, a development of the approach proposed in ref. [20], where the boson system was considered in the framework of the Grand Canonical Ensemble at zero chemical potential. Here, we investigate thermodynamic properties of the interacting particle-antiparticle boson system in the Canonical Ensemble at the conserved isospin density $n_{I}$ and finite temperature $T$, which are now the canonical variables for thermodynamic quantities. To account for the interaction between the bosons we introduce a phenomenological Skyrme-like mean field $U(n)$, which depends only on the total meson density $n$. This mean field rather reflects the presence of other strongly interacting particles in the system, for instance $\rho$ mesons and nucleon-antinucleon pairs at low temperatures or gluons and quark-antiquark pairs at high temperatures, $T>T_{\text {qgp }} \approx 160 \mathrm{MeV}$. Calculations for noninteracting hadron resonance gas show that the particle densities may reach values $(0.1-0.2) \mathrm{fm}^{-3}$ at temperatures $100-$ $160 \mathrm{MeV}$, which are below the deconfinement phase transition, see e.g. refs. [21,22].

In this work in the Canonical Ensemble formulation we calculate thermodynamic characteristics of the non-ideal hot "pion" gas with fixed isospin density $n_{I}=n_{\pi}^{(-)}-n_{\pi}^{(+)}>0$. In Sect. 2 we develop a formalism of the thermodynamic mean-field model [23] for description of a bosonic system of particles and antiparticles which will be used in the presented calculations. In Sect. 3 we introduce a Skyrme-like parametrization of the mean field and calculate corresponding thermodynamic functions. In Sect. 4 we demonstrate the possibility of the Bose condensation when the attractive interaction is "weak". Our conclusions are summarized in Sect. 5 .

\section{The mean-field model for the system of boson particles and antiparticles}

Our consideration in this section is based on the thermodynamic mean-field model which was introduced in refs. [24,25], and then developed in ref. [23]. The basic derivations of the model are present in Appendix.

We limit our consideration to the case where at a fixed temperature the interacting boson particles and boson antiparticles are in the dynamical equilibrium with respect to annihilation and pair-creation processes. The chemical potentials of boson particles $\mu_{p}$ and boson antiparticles $\mu_{\bar{p}}$ are then, have opposite signs:

$$
\mu_{p}=-\mu_{\bar{p}} \equiv \mu .
$$

We are going to consider the system of boson particles and boson antiparticles at conserved isospin number $N_{I} \equiv N^{(-)}-N^{(+)}$, where $N^{(-)}$is the number of boson particles and $N^{(+)}$is the number of boson antiparticles. The total number of mesons in the system is $N=N^{(-)}+N^{(+)}$. Therefore, the Euler relation includes isospin number density only:

$$
\varepsilon+p=T s+\mu n_{I},
$$

where $n_{I}=N_{I} / V$ with $V$ as the volume of the system, and the total particle-number density is $n=n^{(-)}+n^{(+)}$.

Roughly speaking, in such a problem the chemical potential controls the difference of particle and antiparticle numbers $\mu \rightarrow\left(N^{(-)}-N^{(+)}\right)$whereas the total number of particles is controlled by the temperature $T \rightarrow\left(N=N^{(-)}+N^{(+)}\right)$. Indeed, if some amount of particle-antiparticle pairs $M$ has been created additionally to the existing particles $N^{(-)}$and $N^{(+)}$in a closed system, then approximately the same value $\mu$ is in correspondence $\mu \rightarrow$ $\left[\left(N^{(-)}+M\right)-\left(N^{(+)}+M\right)\right]$ but $T^{\prime} \rightarrow\left(N^{(-)}+M+N^{(+)}+M\right)$, where $T^{\prime}>T$. This qualitative consideration indicates the existence of one to one correspondence of independent 
pairs of variables $(T, \mu) \Leftrightarrow\left(N, N_{I}\right)$. Actually, it is an easy task to show that the latter statement is valid in ideal quantum gas of particles and antiparticles. Meanwhile, it is not so simple the rigorous proof of the independence of thermodynamic variables $n$ and $n_{I}$ in a more general case where the mean fields, which depend on these variables, are present in the system (see [26]).

In general the mean field $U$ depends on both independent variables $n, n_{I}$, i.e. $U\left(n, n_{I}\right)$. On the other hand, as proved in [26], the mean field can be separated into $n$-dependent and $n_{I}$-dependent pieces where then, it reads respectively for particles and antiparticles as

$$
\begin{aligned}
& U^{(-)}\left(n, n_{I}\right)=U(n)-U_{I}\left(n_{I}\right), \\
& U^{(+)}\left(n, n_{I}\right)=U(n)+U_{I}\left(n_{I}\right) .
\end{aligned}
$$

These signs in eqs. (3) and (4) are due to odd dependence on the isospin number $n_{I}$.

The total pressure in the two-component system reads

$$
\begin{aligned}
p & =-g T \int \frac{d^{3} k}{(2 \pi)^{3}} \ln \left[1-\exp \left(-\frac{\sqrt{m^{2}+\mathbf{k}^{2}}+U(n)-U_{I}\left(n_{I}\right)-\mu}{T}\right)\right]- \\
& -g T \int \frac{d^{3} k}{(2 \pi)^{3}} \ln \left[1-\exp \left(-\frac{\sqrt{m^{2}+\mathbf{k}^{2}}+U(n)+U_{I}\left(n_{I}\right)+\mu}{T}\right)\right]+P\left(n, n_{I}\right),
\end{aligned}
$$

where $P\left(n, n_{I}\right)$ is the excess pressure. Here and below we adopt the system of units $\bar{h}=c=1$, $k_{B}=1$.

At the first step of investigation we neglect that part of the mean field which depends on isospin density, i.e. we assume $U_{I}\left(n_{I}\right)=0$. Therefore, in this approximation, the excess pressure also depends only on the total particle-number density, $P(n)$.

The thermodynamic consistency of the mean-field model can be obtained by putting in correspondence of two expressions which must coincide in the result. These expressions, which determine the isospin density, read

$$
n_{I}=\left(\frac{\partial p}{\partial \mu}\right)_{T},
$$

where pressure is given by Eq.(5), and

$$
n_{I}=g \int \frac{d^{3} k}{(2 \pi)^{3}}[f(E(k, n), \mu)-f(E(k, n),-\mu)] .
$$

Here $E(k, n)=\omega_{k}+U(n)$ with $\omega_{k}=\sqrt{m^{2}+\boldsymbol{k}^{2}}$ and the Bose-Einstein distribution function reads

$$
f(E, \mu)=\left[\exp \left(\frac{E-\mu}{T}\right)-1\right]^{-1} .
$$

In order the expressions (6) and (7) to coincide in the result the following relation between the mean field and the excess pressure arises

$$
n \frac{\partial U(n)}{\partial n}=\frac{\partial P(n)}{\partial n}
$$

It provides the thermodynamic consistency of the model. When both components of $\pi^{-}-\pi^{+}$ 
system are in the thermal (kinetic) phase the pressure and energy density read

$$
\begin{aligned}
& p=\frac{g}{3} \int \frac{d^{3} k}{(2 \pi)^{3}} \frac{\mathbf{k}^{2}}{\omega_{k}}[f(E(k, n), \mu)+f(E(k, n),-\mu)]+P(n), \\
& \varepsilon=g \int \frac{d^{3} k}{(2 \pi)^{3}} \omega_{k}[f(E(k, n), \mu)+f(E(k, n),-\mu)]+n U(n)-P(n) .
\end{aligned}
$$

\section{Skyrme-like parametrization of the mean field}

The thermodynamic mean-field model has been applied for several physically interesting systems including the hadron-resonance gas [23] and the pionic gas [27]. This approach was extended to the case of a bosonic system at $\mu=0$ which can undergo Bose condensation [20,28]. In the present study a generalized formalism given in section 2 is used to describe the particleantiparticle system of bosons when the isospin density is kept constant. As was mentioned in the previous section the mean field in general case splits into two pieces with dependence on the total particle density $n$ and on the isospin density $n_{I}$, respectively, see eqs. (3) and (4). At the first stage of our investigation we assume that the interaction between particles is described by the Skyrme-like mean field which depends only on the total particle-number density $n$. Loosely speaking we take into account just a strong interaction. So, we assume that the mean field reads

$$
U(n)=-A n+B n^{2},
$$

where $A$ and $B$ are the model parameters, which should be specified. Some additional contribution to the attractive mean field at high temperatures, $(T \propto 100-160 \mathrm{MeV})$, may be provided by other hadrons present in the system, like $\rho$-mesons [29] or baryon-antibaryon pairs [30]. As was mentioned in the introduction, an investigation of the properties of a dense and hot pion gas is well inspired by formation of the medium with low baryon numbers at midrapidity what was proved in the experiments at RHIC and LHC $[3,4]$.

By this reason, in our calculations we consider a general case of $A>0$, to study a bosonic system with both attractive and repulsive contributions to the mean field (12). For the repulsive coefficient $B$ we use a fixed value, obtained from an estimate based on the virial expansion [31], $B=10 m v_{0}^{2}$ with $v_{0}$ equal to four times the proper volume of a particle, i.e. $v_{0}=16 \pi r_{0}^{3} / 3$. In our numerical calculations we take $v_{0}=0.45 \mathrm{fm}^{3}$ that corresponds to a "particle radius" $r_{0} \approx 0.3 \mathrm{fm}$. The numerical calculations will be done for bosons with mass $m=139 \mathrm{MeV}$, which we call "pions". In this case the repulsive coefficient is $B / m=2.025 \mathrm{fm}^{-6}$ and it is kept constant through all present calculations. (For instance, in Ref. [32] authors use the value $B / m=21.6 \mathrm{fm}^{-6}$.) At the same time the coefficient $A$, which determines the intensity of attraction of the mean field (12), will be varied. It is advisable to parameterize the coefficient $A$. We are going to do this with making use of solutions of equation $U(n)+m=0$, similar to parametrization adopted in refs. [20,28]. For the given mean field (12) there are two roots of this equation $\left(n_{1,2}=\left(A \mp \sqrt{A^{2}-4 m B}\right) / 2 B\right)$

$$
n_{1}=\sqrt{\frac{m}{B}}\left(\kappa-\sqrt{\kappa^{2}-1}\right), \quad n_{2}=\sqrt{\frac{m}{B}}\left(\kappa+\sqrt{\kappa^{2}-1}\right),
$$

where

$$
\kappa \equiv \frac{A}{2 \sqrt{m B}}
$$


Then, one can parameterize the attraction coefficient as $A=\kappa A_{\mathrm{c}}$ with $A_{\mathrm{c}}=2 \sqrt{m B}$. As we show further, the dimensionless parameter $\kappa$ is a scaling parameter of the model, i.e. when the isospin density is a constant the parameter $\kappa$ determines the phase structure of the system. As it is seen from eq. (13) for the values of parameter $\kappa<1$ there are no real roots. The critical value of parameter $A$ is obtained when both roots coincide, i.e. when $\kappa=1$, then $A=A_{\mathrm{c}}=2 \sqrt{m B}$. We consider two intervals of the parameter $\kappa$. 1) First interval corresponds to $\kappa \leq 1$, there are no real roots of equation $U(n)+m=0$. We associate these values of $\kappa$ with a "weak" attractive interaction and in the present study we consider variations of the attractive coefficient $A$ just for values of $\kappa$ from this interval. 2) Second interval corresponds to $\kappa>1$, there are two real roots of equation $U(n)+m=0$. We associate this interval with a "strong" attractive interaction. This case will be considered elsewhere.

If one assumes a possibility of the Bose-Einstein condensation in the two-component system, then it is instructive to classify a phase structure of the system in accordance with two basic combinations which determine for the "weak" attraction the different thermodynamic states: (i) Both components, or the boson particles and boson antiparticles, i.e. $\pi^{-}$and $\pi^{+}$, are in the thermal (kinetic) phase; (ii) Particles $\left(\pi^{-}\right)$are in the condensate phase and antiparticles $\left(\pi^{+}\right)$are in the thermal (kinetic) phase - this combination can be named as the "cross" state.

It is necessary to note, that expression "particles are in the condensate phase" is, of course, a conventional one, because in the essence it is a mixture phase, where at a fixed temperature some fraction of particles, i.e. a fraction of $\pi^{-}$-mesons, is in thermal states with momentum $|\boldsymbol{k}|>0$ and other fraction of this $\pi^{-}$-component belongs to the Bose-Einstein condensate, where all $\pi^{-}$-mesons have zero momentum, $\boldsymbol{k}=0$.

We are going now to consider these basic thermodynamic states of the system using the mean field (12).

\section{Thermodynamic properties of the bosonic particle-antiparticle system at "weak attraction"}

In the mean-field approach the behavior of the particle-antiparticle bosonic system in thermal (kinetic) phase is determined by the set of two transcendental equations (we keep $n_{I}=$ const)

$$
\begin{aligned}
n & =\int \frac{d^{3} k}{(2 \pi)^{3}}[f(E(k, n), \mu)+f(E(k, n),-\mu)], \\
n_{I} & =\int \frac{d^{3} k}{(2 \pi)^{3}}[f(E(k, n), \mu)-f(E(k, n),-\mu)],
\end{aligned}
$$

where the Bose-Einstein distribution function $f(E, \mu)$ is defined in (8) and $E(k, n)=\omega_{k}+$ $U(n)$. Equations (15)-(16) should be solved selfconsistently with respect to $n$ and $\mu$ for a given temperature $T$ with account for $n_{I}=$ const. In the present we consider bosonical system in the Canonical Ensemble, where the independent canonical variables are $T$ and $n_{I}$, particles spin equal to zero. In this approach the chemical potential $\mu$ is a thermodynamic variable which depends on the canonical variables, i.e. $\mu\left(T, n_{I}\right)$.

In case of the cross state, when the particles, i.e. $\pi^{-}$-mesons, are in the condensate phase and antiparticles are still in the thermal (kinetic) phase, eqs. (15), (16) should be generalized to include condensate component $n_{\text {cond }}^{(-)}$. Besides this we should take into account that the particles ( $\pi^{-}$or high-density component) can be in condensed state just under the necessary condition

$$
U(n)-\mu=-m \text {. }
$$


During decreasing of temperature from high values, where both $\pi^{-}$and $\pi^{+}$are in the thermal phase, the density of $\pi^{-}$-component $n^{(-)}(T, \mu)$ (high-density component) achieves first the critical curve at temperature $T_{\mathrm{c}}^{(-)}$, where condition (17) is valid. This means that the curve $n_{\lim }^{(\text {id) }}(T)$, which is defined as

$$
n_{\lim }^{(\mathrm{id})}(T)=\left.\int \frac{d^{3} k}{(2 \pi)^{3}} f\left(\omega_{k}, \mu\right)\right|_{\mu=m}
$$

is the critical curve for $\pi^{-}$-mesons or for high-density component. Here $f\left(\omega_{k}, \mu\right)$ is the BoseEinstein distribution function defined in (8). As we see function (18) represents the maximal density of thermal (kinetic) boson particles of the ideal gas at temperature $T$ when $\mu=m$. Hence, we obtain that the critical curve in the mean-field approach under consideration for the boson particles coincides with the critical curve for the ideal gas.

With account for eqs. (17) and (18) we write the generalization of the set of eqs. (15), (16)

$$
\begin{aligned}
n & =n_{\text {cond }}^{(-)}(T)+n_{\lim }^{(\mathrm{id})}(T)+\int \frac{d^{3} k}{(2 \pi)^{3}} f(E(k, n),-\mu), \\
n_{I} & =n_{\text {cond }}^{(-)}(T)+n_{\lim }^{(\mathrm{id})}(T)-\int \frac{d^{3} k}{(2 \pi)^{3}} f(E(k, n),-\mu) ;
\end{aligned}
$$

Meanwhile, because of relation (17) between the mean field and chemical potential, i.e. $E(k, n)-$ $\mu=\omega_{k}-m$, this set of equations can be reduced just to one equation with respect to $n^{(+)}$and it reads

$$
n^{(+)}=\left.\int \frac{d^{3} k}{(2 \pi)^{3}} f(E(k, n),-\mu)\right|_{\mu=U(n)+m} \quad \text { with } \quad E(k, n)=\omega_{k}+U\left(2 n^{(+)}+n_{I}\right) .
$$

Solution of eq. (21) for temperatures $T$ from the interval $T<T_{\mathrm{c}}^{(-)}$provides the density $n^{(+)}(T)$ of $\pi^{+}$-mesons.

One can see from eqs. (19), (20) that the particle density $n^{(+)}$is provided only by thermal (kinetic) antiparticles ( $\pi^{+}$-mesons). Whereas, the density $n^{(-)}$of $\pi^{-}$-mesons is provided by two fractions: (1) the condensed particles $\left(\pi^{-}\right.$-mesons at $\left.\boldsymbol{k}=0\right)$ with the particle-number density $n_{\text {cond }}^{(-)}(T)$, and (2) thermal $\pi^{-}$-mesons at $|\boldsymbol{k}|>0$ with the particle-number density $n_{\lim }^{(\mathrm{id})}(T)$. The particle-density sum rule for these phase of $\pi^{-}$-mesons in the interval $T<T_{\mathrm{c}}^{(-)}$reads

$$
n^{(-)}=n_{\text {cond }}^{(-)}(T)+n_{\lim }^{(\mathrm{id})}(T) .
$$

\subsection{Numerical calculations}

At high temperatures, i.e. $T \geq T_{\mathrm{c}}^{(-)}$, both components of the bosonic particle-antiparticle system are in the thermal phase and thermodynamic properties of the system are determined by the set of eqs. (15) and (16). Solving this set for given values $T$ and $n_{I}$ we obtain the functions $\mu\left(T, n_{I}\right)$ and $n\left(T, n_{I}\right)$ and then other thermodynamic quantities.

When we decrease temperature, after crossing the value $T=T_{\mathrm{c}}^{(-)}$the particles which belong to the high-density component (or $\pi^{-}$-mesons) start to "drop down" into the condensate state, which is characterized by the value of momentum $\boldsymbol{k}=0$. In the limit, when $T=0$, all particles of the high-density component $n^{(-)}$, i.e. $\pi^{-}$-mesons, are in condensed state. At the 

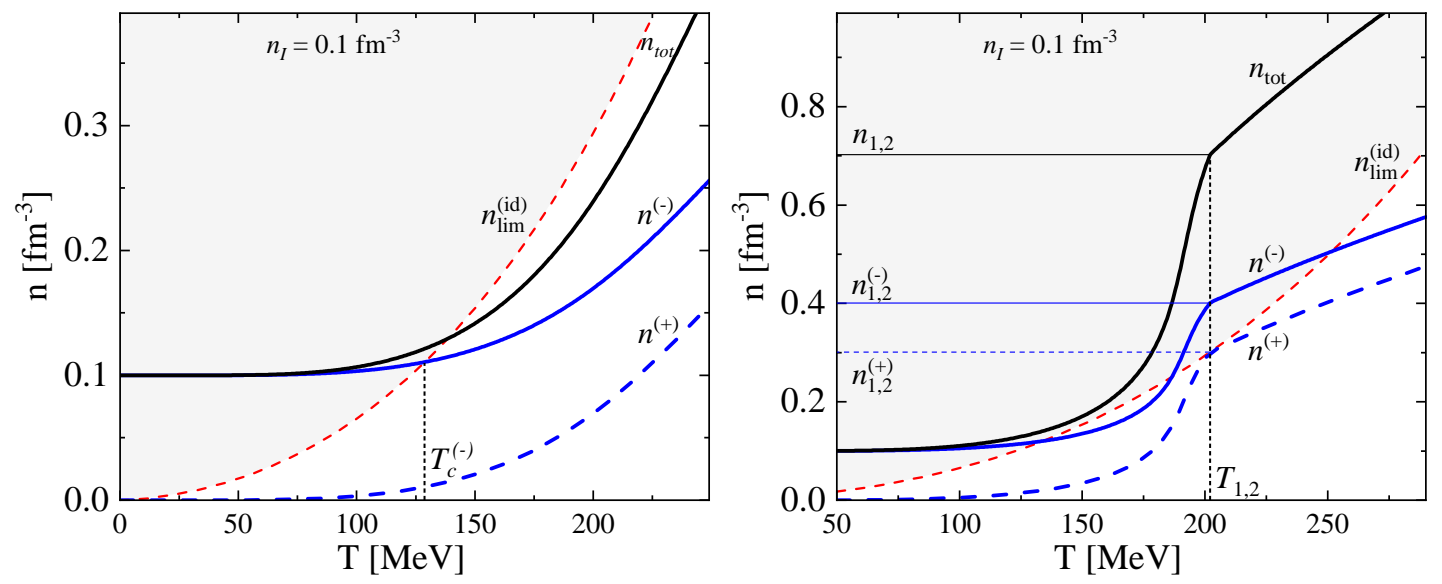

Fig. 1. Left panel: The particle-number densities $n^{(+)}, n^{(-)}$and $n_{\text {tot }}=n^{(+)}+n^{(-)}$versus temperature for the interacting $\pi^{+}-\pi^{-}$pion gas in the mean-field Skyrme-like model. The total isotope-spin density (which kept constant) is $n_{\mathrm{I}}=0.1 \mathrm{fm}^{-3}$ and attraction parameter is $\kappa=0.5$. The maximal density $n_{\mathrm{lim}}^{\text {(id) }}$ of the ideal gas of thermal pions at $\mu=m_{\pi}$ depicted as red dashed line and the dashed area indicates the phase of condensed particles. Diamond shows the temperature $T_{\mathrm{c}}^{(-)}$of bosonic condensation of $\pi^{-}$pions. Right panel: The same as in left panel but at parameter $\kappa=1.0$, where $n_{1}=n_{2} \equiv n_{1,2}$ and $T_{1}=T_{2} \equiv T_{1,2}$.

same time, the particles of the low-density component or $\pi^{+}$-mesons being in the thermal phase lose the density $n^{(+)}$with decrease of temperature and it becomes rigorously zero at $T=0$. For the temperature interval $T<T_{\mathrm{c}}^{(-)}$equations (15), (16) should be generalized and now thermodynamic properties of the system are determined by eq. (21), where we take into account that $\mu=-U(n)+m$ for all temperatures of this interval unless the high-density component $n^{(-)}$is in condensed state. Otherwise it is necessary to solve the set of eqs. (15) and (16) for the region where $n^{(-)}$appears again in the thermal (kinetic) phase.

For parameters $n_{\mathrm{I}}=0.1 \mathrm{fm}^{-3}, \kappa=0.5$ and $\kappa=1.0$ we solve the set of eqs. (15), (16) for the thermal phase and eq. (21) for the "cross" thermodynamic state. The behavior of the density $n^{(+)}$of $\pi^{+}$-mesons and the density $n^{(-)}$of $\pi^{-}$-mesons are depicted in Fig. 1. In this figure we depicted as well the behavior of the total meson density $n=n^{(+)}+n^{(-)}$as functions of the temperature (in the figure field it is notated as $n_{\text {tot }}$ ).

Analyzing the behavior of the condensate creation (see Fig. 1) it is necessary to note, that just high-density component of the particle-antiparticle gas undergoes the phase transition to the Bose-Einstein condensate. If we apply our consideration to pion gas with $n_{I}=n_{\pi}^{(-)}-n_{\pi}^{(+)}>0$ this means that $\pi^{-}$-component undergoes the phase transition to the Bose-Einstein condensate and the low-density component or $\pi^{+}$mesons exist only in the thermal phase for whole range of temperatures. Hence, it makes sense to look for the Bose-Einstein condensate of $\pi^{-}$mesons only in an experiment, for instance in heavy-ion collisions.

Equation (19) can be used to determine the critical temperature $T_{\mathrm{c}}^{(-)}$. Indeed, let us take into account that at the crossing point with the critical curve the density of condensate is zero so far, $n_{\text {cond }}^{(-)}\left(T_{\mathrm{c}}^{(-)}\right)=0$, and the density of thermal $\pi^{-}$particles becomes equal to $n^{(-)}\left(T_{\mathrm{c}}^{(-)}\right)=$ $n_{\lim }^{(\mathrm{id})}\left(T_{\mathrm{c}}^{(-)}\right)$. Then, at this temperature $T=T_{\mathrm{c}}^{(-)}$on the 1.h.s. of eq. (19) we have $n=$ $2 n_{\lim }^{\text {(id) }}\left(T_{\mathrm{c}}^{(-)}\right)-n_{I}$, and now at this temperature point on the critical curve eq. (19) with respect to $T$ reads as:

$$
n_{\lim }^{(\mathrm{id})}(T)-n_{I}=\left.\int \frac{d^{3} k}{(2 \pi)^{3}} f(E(k, n),-\mu)\right|_{\mu=U(n)+m} .
$$



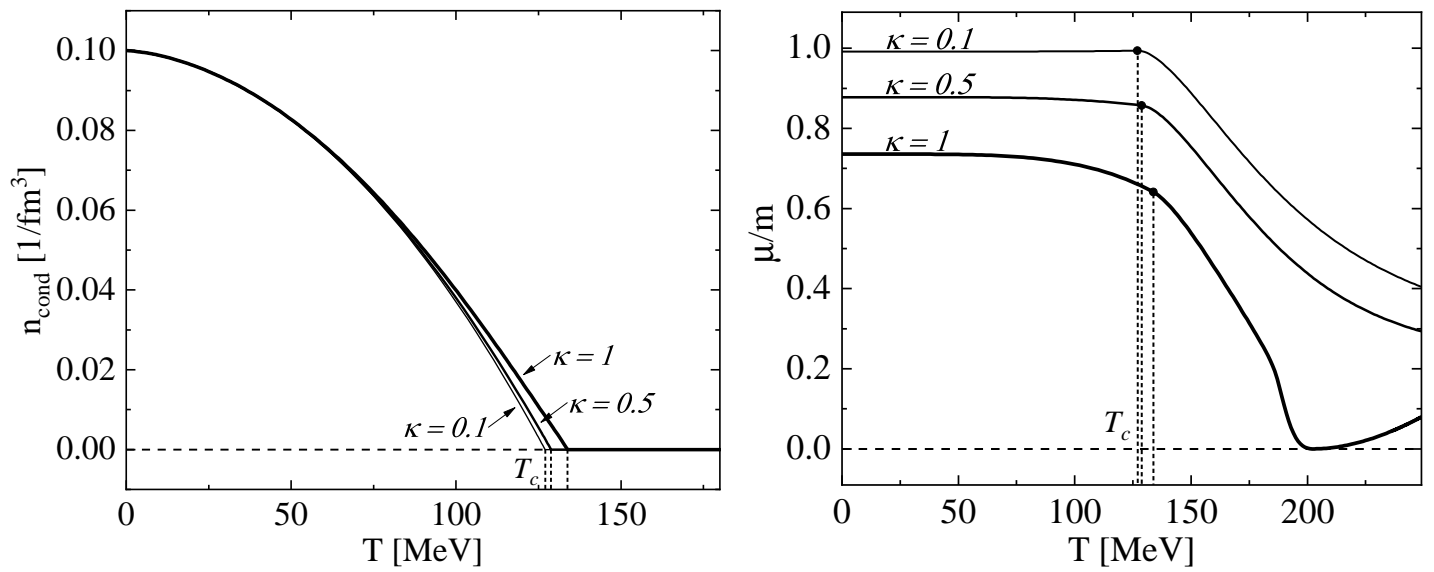

Fig. 2. Left panel: The density of condensate versus temperature in the particle-antiparticle selfinteracting system when the isospin density is kept constant, $n_{\mathrm{I}}=0.1 \mathrm{fm}^{-3}$. The curves are marked by the attraction parameter $\kappa$. Right panel: The chemical potential versus temperature at the values of attraction parameter $\kappa=0.1,0.5,1.0$. The open circles indicate the points, which correspond to the critical temperature $T_{\mathrm{c}}^{(-)}$, appropriate for corresponding parameter $\kappa$. We can assume that approximately $T_{\mathrm{c}} \approx T_{\mathrm{c}}^{(-)}$, where the phase transition to the condensate phase of the high-density component $n^{(-)}$occurs.

where $E(k, n)=\omega_{k}+U(n)$ and $n=2 n_{\text {lim }}^{\text {(id) }}-n_{I}$. Solving eq. (23) at $n_{I}=0.1 \mathrm{fm}^{-3}$, for $\kappa=0.5$ and $\kappa=1.0$ we obtained $T_{\mathrm{c}}^{(-)}=128.8 \mathrm{MeV}$ and $T_{\mathrm{c}}^{(-)}=251 \mathrm{MeV}$, respectively. These results are depicted in Fig. 1 on left and right panels, respectively.

It turns out that the temperature $T_{\mathrm{c}}^{(-)}$determines the phase transition to BEC for whole pion system because the antiparticles ( $\pi^{+}$-mesons) from the low-density component $n^{(+)}(T)$ are completely in thermal state for all temperatures and thus, the condensate is created just by the particles of high-density component $n^{(-)}(T)$. Then, the total density of condensate in the two-component pion system is created by $\pi^{-}$-mesons only, i.e. $n_{\text {cond }}=n_{\text {cond }}^{(-)}$, and this particlenumber density plays the role of the order parameter. The condensate density as function of temperature obtained in the framework of our model for three values of the attraction parameter, $\kappa=0.1,0.5,1.0$, at $n_{\mathrm{I}}=0.1 \mathrm{fm}^{-3}$, is depicted in Fig. 2, left panel. We record a very small discrepancy of the critical temperature $T_{\mathrm{c}}^{(-)}$on variations of the attraction parameter $\kappa$, the difference is not more than $2 \mathrm{MeV}$. Then, one can use the following approximation

$$
T_{\mathrm{c}}=\left\langle T_{\mathrm{c}}^{(-)}\right\rangle
$$

what gives for the particular choice of parameters $T_{\mathrm{c}} \approx 129 \mathrm{MeV}$. This is a temperature of the second order phase transition which "signals" of the creation of condensate when temperature decreases and crosses the value $T=T_{\mathrm{c}}$. Note, the critical temperature $T_{\mathrm{c}}$ does not depend virtually on the attraction parameter $A$ of the mean field (12).

The dependence of the chemical potential on temperature is depicted in Fig. 2 on the right panel for three values of the attraction parameter, $\kappa=0.1,0.5,1.0$. First of all, we notice that the chemical potential is almost independent of temperature when condensate exists in the system, i.e. in the interval $0<T \leq T_{\mathrm{c}}$. Value of $\mu$ changes from $0.99 m_{\pi}$ at small attraction, $\kappa=0.1$, to $\mu=0.74 m_{\pi}$ for the critical attraction parameter $\kappa=1.0$. Hence, for $0.1 \leq \kappa \leq 1.0$ the chemical potential is in the range $103 \leq \mu \leq 138 \mathrm{MeV}$. It is intriguing to remind that already first attempts to fit the $p_{\mathrm{T}}$ spectra of $\pi^{-}$-mesons in $\mathrm{O}+\mathrm{Au}$ collisions at $200 \mathrm{AGeV} /$ nucleon (at midrapidity) by the ideal-gas Bose-Einstein distribution results in the values $\mu \approx 126 \mathrm{MeV}$, 

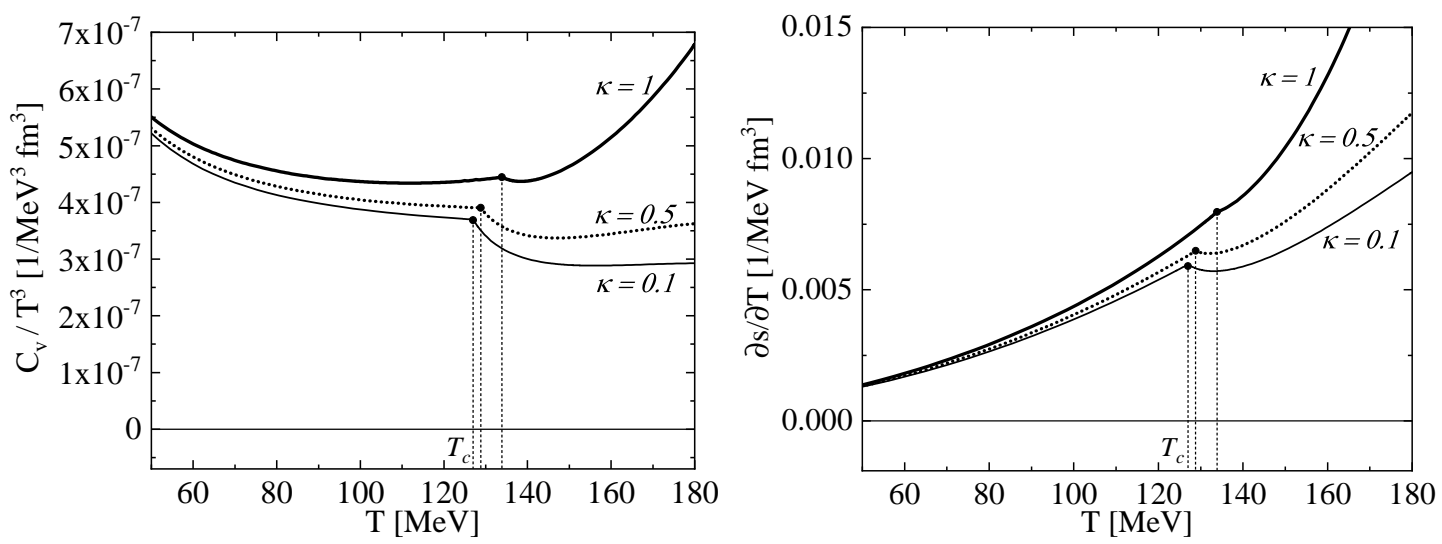

Fig. 3. Left panel: Specific heat normalized to $T^{3}$ versus temperature in the selfinteracting $\pi^{-}-\pi^{+}$-system when the isospin density is kept constant, $n_{\mathrm{I}}=0.1 \mathrm{fm}^{-3}$. The curves are marked by the attraction parameter $\kappa$. Right panel: The derivative of the entropy density with respect to temperature for the same system as on the left panel.

$T \approx 167 \mathrm{MeV}$ and in $\mathrm{S}+\mathrm{S}$ collisions at $200 \mathrm{AGeV} /$ nucleon it results in the values $\mu \approx 118 \mathrm{MeV}$, $T \approx 164 \mathrm{MeV}$ [33]. So, the fit of data required the pion chemical potential in the range $\mu \approx$ $115-130 \mathrm{MeV}$ what we can just formally compare with the values of the chemical potential obtained in our model.

The derivative of $\mu(T)$ with respect to temperature has a jump in the points, which is indicated in Fig. 2 on right panel as open circles. These points on the curves $\mu(T)$ correspond to $T_{\mathrm{c}}^{(-)}$, which values differ from one another not more than $\Delta T=2 \mathrm{MeV}$. As we concluded before, this is the temperature of phase transition, see eq. (24), which practically does not depend on intensity of attraction. To prove that this is indeed a phase transition of the second order, we first consider the specific heat $C_{\mathrm{V}}$ in the system under consideration

$$
C_{\mathrm{V}}=-T \frac{\partial^{2} f}{\partial T^{2}}=T \frac{\partial s}{\partial T}
$$

where $f\left(T, n_{I}\right)$ is the density of the free energy and $s\left(T, n_{I}\right)$ is the entropy density. The density of the free energy reads

$$
f=-p\left(T, n_{I}\right)+n_{I} \mu\left(T, n_{I}\right) .
$$

Having solved eqs. (15), (16) in accordance with eq. (10) one can calculate pressure for the case when particles and antiparticles are both in the thermal phase. Then, the density of the free energy looks like

$$
f=n_{I} \mu\left(T, n_{I}\right)-\frac{1}{3} \int \frac{d^{3} k}{(2 \pi)^{3}} \frac{\mathbf{k}^{2}}{\omega_{k}}[f(E(k, n), \mu)+f(E(k, n),-\mu)]-P(n),
$$

where functions $n\left(T, n_{I}\right)$ and $\mu\left(T, n_{I}\right)$ are known. Here, we use the excess pressure $P(n)$, which is obtained by integrating eq. (9) for the Skyrme-like parametrization of the mean field (12) with taking into account that $P(n=0)=0$,

$$
P(n)=-\frac{A}{2} n^{2}+\frac{2 B}{3} n^{3},
$$

(Remind in the present consideration we neglect $P_{I}\left(n_{I}\right)$ ). 
For temperatures less than $T_{\mathrm{c}}$ when the high-density component of the pion gas ( $\pi^{-}$mesons) is in the condensate phase and low-density component ( $\pi^{+}$mesons) is in the thermal phase, the density of the free energy reads $(\mu=U(n)+m$ as in eq. (21)):

$$
\begin{aligned}
f= & n_{I}[U(n)+m]-\left.\frac{1}{3} \int \frac{d^{3} k}{(2 \pi)^{3}} \frac{\mathbf{k}^{2}}{\omega_{k}} f\left(\omega_{k}, \mu\right)\right|_{\mu=m} \\
& -\left.\frac{1}{3} \int \frac{d^{3} k}{(2 \pi)^{3}} \frac{\mathbf{k}^{2}}{\omega_{k}} f(E(k, n),-\mu)\right|_{\mu=U(n)+m}-P(n) .
\end{aligned}
$$

Here the total pion density is $n=2 n^{(+)}+n_{I}$ and $E(k, n)=\omega_{k}+U(n)$, where $n^{(+)}\left(T, n_{I}\right)$ is solution of eq. (21). Using the density of the free energy (27) to the right of $T_{\mathrm{c}}$ and (29) to the left of $T_{\mathrm{c}}$, respectively, we calculate the specific heat normalized to $T^{3}$, as function of temperature at $n_{\mathrm{I}}=0.1 \mathrm{fm}^{-3}$ for three values of the attraction parameter $\kappa=0.1,0.5,1.0$. These dependencies are depicted in Fig. 3 on the left panel. It is obvious that dependence of the specific heat on temperature is a continuous function but the derivative of this function has a finite gap. To be convinced on that we depicted separately in Fig. 3 on the right panel the temperature dependence of the derivative of the entropy density $s_{T}=\partial s / \partial T$ for the same set of parameters as on the left panel. Evidently seen is the same break of the derivatives of the functions $s_{T}(T)$, which exhibits a discontinuity of the derivative of third order of the density of the free energy. It has long been known (see ref. [34] for confirmation) that the Bose-Einstein condensation is indeed a third-order phase transition according to the first classification of general types of transitions between phases of matter, introduced by Paul Ehrenfest in $1933[35,36]$. Therefore, the obtained temperature $T_{\mathrm{c}}$ is really the temperature of the phase transition of the second order (according to modern terminology) and the density of condensate $n_{\text {cond }}=n_{\text {cond }}^{(-)}$ provided by $\pi^{-}$mesons is the order parameter.

\section{Concluding remarks}

In this paper we have presented a thermodynamically consistent method to describe at finite temperatures a dense bosonic system which consists of interacting particles and antiparticles at a fixed isospin density $n_{I}$. We considered the system of meson particles with $m=m_{\pi}$ and zero spin, which we named conventionally as "pions" because the charged $\pi$-mesons are the lightest nuclear particle and the lightest hadrons that couple to the isospin chemical potential. It was shown that in the particle-antiparticle meson system, where the isospin density $n_{I}$ is conserved, the Bose-Einstein condensate exists in the system starting from zero temperature what is a result of the phase transition of the second order which occurs at the temperature $T_{\mathrm{c}}$ when temperature decreases. ${ }^{1}$ This statement is in contrast to the conclusion given in Refs. [20,28,32,37], where the system with zero chemical potential, $\mu=0$, was investigated. Indeed, in [20, 28, 32, 37] was shown that just in case the attractive mean field is strong enough the multi-boson system undergoes the phase transition of the first order and as a result it develops the Bose condensate starting from a finite temperature.

It was obtained that independently of parameters of the mean field the multi-boson system develops the Bose condensate for particles of high-density component only. This means that in the pion gas, where $n_{I}=n_{\pi}^{(-)}-n_{\pi}^{(+)}>0$, the $\pi^{-}$mesons only undergo the phase transition to the Bose-Einstein condensate. At the same time, the $\pi^{+}$mesons exist only in the thermal phase

\footnotetext{
${ }^{1}$ Note, the chiral perturbation theory predicts that transition between the vacuum and the BEC state is of the second order with universality class $O(2)[13]$.
} 
for whole range of temperatures. Then, for the experimental efforts it makes sense to look for the Bose condensate, which is created just by $\pi^{-}$mesons.

It was shown that the dimensionless quantity $\kappa=A / 2 \sqrt{m B}$, which itself is a combination of the parameters of the mean field, $U(n)=-A n+B n^{2}$, and particle mass, is the scaling parameter of the model. The parameter $\kappa$ determines the different possible phase scenarios which occur in the particle-antiparticle boson system. The attractive coefficient $A=\kappa A_{\mathrm{c}}$, where $A_{\mathrm{c}} \equiv 2 \sqrt{m B}$, was parameterized by the $\kappa$ with $\kappa=1$ as a critical value, which separates the regime of "weak attraction" $(\kappa \leq 1)$ from the regime of "strong attraction" $(\kappa>1)$. In this paper, we have considered only the case of "weak attraction".

Description of thermodynamic properties of the system was performed employing the Canonical Ensemble formulation, where the chemical potential $\mu$ is a thermodynamic quantity which depends on the canonical variables $\left(T, n_{I}\right)$. It was obtained dependencies of chemical potential on temperature for different attraction parameters $\kappa$ which show that $\mu \approx$ const in the "condensate" interval of temperatures $0 \leq T \leq T_{\mathrm{c}}$, where these constant values essentially depend on the intensity of attraction. Meanwhile the temperature $T_{\mathrm{c}}^{(-)}$of the phase transition to BEC of $\pi^{-}$(high-density component) exhibits very weak dependence on $\kappa$, as it is evidently seen in Fig. 2, left panel. For wide range of the values of $\kappa$, from 0.1 to 1.0, these critical temperatures differ from one another not more than $2 \mathrm{MeV}$, this inspires the introduction of the mean value $T_{\mathrm{c}}=\left\langle T_{\mathrm{c}}^{(-)}\right\rangle$of the phase transition to BEC.

The results obtained are in correspondence with known peculiar property of the ideal Bose gas: the Bose-Einstein condensation represents the phase transition of third order or a discontinuity of the derivative of the specific heat [34]. In the framework of the present model we obtained that in the same way the derivative of the specific heat undergoes a break at the temperature $T_{\mathfrak{c}}$, as it is evidently seen on the left panel in Fig. 3. A discontinuity of the third derivative of the free energy one can see on the right panel in Fig. 3 as a break of the derivative of the entropy density at the temperature $T_{\mathrm{c}}$.

The role of neutral pions is left beyond the scope of the present paper. The present analysis can be improved by addressing these issue in more detail and also by generalizing the calculation to nonzero contribution to the mean field which depends on $n_{I}$. Authors plan to consider these problems elsewhere.

\section{Acknowledgements}

We thank H. Stoecker for support and I. Mishustin and V. Vovchenko for reading the manuscript and making the valuable remarks. The work of D. A. is supported by the National Academy of Sciences of Ukraine by its priority project "Fundamental properties of the matter in the relativistic collisions of nuclei and in the early Universe" (No. 0120U100935). The work of D. Zh. is supported by the Program "The structure and dynamics of statistical and quantum-field systems" of the Department of Physics and Astronomy of NAS of Ukraine.

\section{A. Self-consistent statistical mechanics in mean-field approach}

First we shortly remind the basics of the thermodynamical mean-field model which was introduced in ref. [23], see more details in [24,25].

Let us consider the system of interacting particles from general thermodynamic point of view. One can describe such a system in terms of the free energy density $\phi(n, T)$, which depends on particle density $n$ and temperature $T$. The free energy density (FED) is related to other 
thermodynamical quantities as follows

$$
\begin{aligned}
& \phi(n, T)=\varepsilon(n, T)-T s(n, T), \\
& \phi(n, T)=n \mu(n, T)-p(n, T),
\end{aligned}
$$

where $\varepsilon(n, T)$ is the energy density and $p(n, T)$ is the pressure. Two quantities $\mu(n, T)$ (the chemical potential) and $s(n, T)$ (the entropy density) are given as partial derivatives with respect to independent variables $(n, T)$

$$
\mu=\left(\frac{\partial \phi}{\partial n}\right)_{T}, \quad s=-\left(\frac{\partial \phi}{\partial T}\right)_{n} .
$$

Very generally, for a system of interacting particles the FED can be written as a sum of free and interacting contributions

$$
\phi(n, T)=\phi_{0}(n, T)+\phi_{\text {int }}(n, T),
$$

where $\phi_{0}$ is the FED of the noninteracting system. The chemical potential can also be splitted into "free" and "interacting" pieces. In accordance with eq. (32) we obtain

$$
\mu=\mu_{0}+\left(\frac{\partial \phi_{\text {int }}}{\partial n}\right)_{T}, \quad \text { where } \quad \mu_{0} \equiv\left(\frac{\partial \phi_{0}}{\partial n}\right)_{T} .
$$

Further, taking into account eqs. (31), (33) and (34) one can represent the pressure in the following form

$$
p=n \mu(n, T)-\phi(n, T)=p_{0}(n, T)+n\left(\frac{\partial \phi_{\mathrm{int}}}{\partial n}\right)_{T}-\phi_{\mathrm{int}},
$$

where

$$
p_{0}(n, T)=n \mu_{0}(n, T)-\phi_{0}(n, T) .
$$

One can put this expression in correspondence to the pressure of the ideal gas $\widetilde{p}_{0}$ calculated in the grand canonical ensemble for the same values $T$ and $\mu_{0}$ as they are taken in (36)

$$
\widetilde{p}_{0}\left(T, \mu_{0}\right)=\frac{g}{3} \int \frac{d^{3} k}{(2 \pi)^{3}} \frac{\boldsymbol{k}^{2}}{\omega_{k}} f\left(\omega_{k}, \mu_{0}\right),
$$

where $g$ is the degeneracy factor, $f\left(\boldsymbol{k} ; T, \mu_{0}\right)$ is the ideal gas distribution function (the Boltzmann, Fermi-Dirac or Bose-Einstein one), which depends on temperature and ideal chemical potential $\mu_{0}$, and $\omega_{k}=\sqrt{m^{2}+\boldsymbol{k}^{2}}$.

Now we introduce the following important notations:

$$
\begin{aligned}
U(n, T) & =\left[\frac{\partial \phi_{\mathrm{int}}(n, T)}{\partial n}\right]_{T}, \\
P_{\mathrm{ex}}(n, T) & =n\left[\frac{\partial \phi_{\mathrm{int}}(n, T)}{\partial n}\right]_{T}-\phi_{\mathrm{int}}(n, T) .
\end{aligned}
$$


One can immediately see that these quantities are related as

$$
n \frac{\partial U(n, T)}{\partial n}=\frac{\partial P_{\mathrm{ex}}(n, T)}{\partial n} .
$$

By subtracting eq. (39) from eq. (35) one can rewrite the total pressure as

$$
p=p_{0}(n, T)+P_{\mathrm{ex}}(n, T) .
$$

Evidently, if one defines $p_{0}(n, T)$ as the pressure of the ideal gas, then, the quantity $P_{\mathrm{ex}}(n, T)$ should be regarded as an excess pressure, which is due to the interaction between particles.

Next, in our evaluations of the thermodynamic quantities of the interacting system we would like to use formula (37) for the pressure of the ideal gas. In the canonical ensemble the independent variables are $n$ and $T$, whereas in the grand canonical ensemble they are $\mu$ and $T$. Hence, it is necessary to express the free chemical potential $\mu_{0}$ through these variables. One can do this by substituting eq. (38) into eq. (34), thus obtaining

$$
\mu=\mu_{0}+U(n, T) .
$$

It is convenient to introduce also the single-particle energy for interacting particles

$$
E(\boldsymbol{k}, n)=\sqrt{m^{2}+\boldsymbol{k}^{2}}+U(n) .
$$

In the grand canonical ensemble we treat the particle density $n$ as $n(\mu, T)$, and as a result, the pressure of interacting particles can be expressed as

$$
p(T, \mu)=\frac{g}{3} \int \frac{d^{3} k}{(2 \pi)^{3}} \frac{\boldsymbol{k}^{2}}{\sqrt{m^{2}+\boldsymbol{k}^{2}}} f(E(\boldsymbol{k}, n), \mu)+P_{\mathrm{ex}}(n, T),
$$

where

$$
f(E, \mu)=\left\{\exp \left[\frac{E-\mu}{T}\right]+a\right\}^{-1}
$$

with $a=+1$ for fermions, $a=-1$ for bosons and $a=0$ for the Boltzmann statistics.

In a homogeneous system, where the thermodynamic potential can be expressed as $\Omega(T, \mu, V)=$ $-p(T, \mu) V$, the particle density reads $n(T, \mu)=\partial p(T, \mu) / \partial \mu$. Then, with the help of relations (40) and (44) we obtain the standard relation

$$
n=g \int \frac{d^{3} k}{(2 \pi)^{3}} f(\boldsymbol{k} ; T, \mu) .
$$

Since the distribution function $f(\boldsymbol{k} ; T, \mu)$ is itself a function of $n$, this expression is not a formula for particle density $n$, but an equation to be solved in a selfconsistent way for every point of the $(T, \mu)$ plane. The solution will result in the explicit dependence $n=n(T, \mu)$, which in general differs from the ideal gas expression, $n_{0}\left(T, \mu_{0}\right)$. Using $s=\partial p / \partial T$ and the Euler relation, 
$\varepsilon+p=T s+\mu n$, one obtains for the energy density

$$
\begin{aligned}
\varepsilon(T, \mu)= & g \int \frac{d^{3} k}{(2 \pi)^{3}} \sqrt{m^{2}+\boldsymbol{k}^{2}} f(\boldsymbol{k} ; T, \mu)+n U(n, T)-P_{\mathrm{ex}}(n, T)+ \\
& +T\left\{\left[\frac{\partial P_{\mathrm{ex}}(n, T)}{\partial T}\right]_{n}-n\left[\frac{\partial U(n, T)}{\partial T}\right]_{n}\right\} .
\end{aligned}
$$

This type of approach is widely used in relativistic mean field models of nuclear matter [38], where the nucleons interact with a scalar field $\varphi$ (attraction) and a vector field $V_{\mu}$ (repulsion). In the homogeneous static system only the "time" component of the vector field $V_{0}$ survives. In our notations it is equal to the mean field $U(n, T)$.

\section{References}

1. Busza, W. Heavy Ion Collisions: The Big Picture and the Big Questions/ W. Busza, K. Rajagopal and W. Van Der Schee// Annual Review of Nuclear and Particle Science 68, 339 (2018).

2. Bzdak, A. Mapping the Phases of Quantum Chromodynamics with Beam Energy Scan/ A. Bzdak, S. Esumi, V. Koch, J. Liao, M. Stephanov, and N. Xu// Physics Reports 853, 1-87 (2020).

3. Adamczyk, L. Bulk properties of the medium produced in relativistic heavy-ion collisions from the beam energy scan program/ L. Adamczyk et al. [STAR Collab.]// Phys. Rev. C 96, 044904 (2017).

4. Abelev, B. Pion, kaon, and protonproduction in central $\mathrm{Pb}-\mathrm{Pb}$ collisions at $\sqrt{s_{N N}}=$ 2.76 TeV/ B. Abelev et al. [ALICE Collab.]// Phys. Rev. Lett. 109, 252301 (2012).

5. Migdal, A.B. Stability of Vacuum and Limiting Fields/ A.B. Migdal// Sov. Phys. JETP 34(6), 1184 (1972).

6. Migdal, A.B. The pion spectrum in nuclear matter and pion condensation/ A.B. Migdal, O.A. Markin, I.N. Mishustin// Sov. Phys. JETP 39(2), 212 (1974).

7. Migdal, A.B. Pion fields in nuclear matter/ A.B. Migdal// Rev. Mod. Phys. 50, 107 (1978).

8. Migdal, A.B. Pion degrees of freedom in nuclear matter/ A.B. Migdal, E. Saperstein, M. Troitsky, and D. Voskresensky// Phys. Rept. 192, 179 (1990).

9. Anselm, A. Production of classical pion field in heavy ion high energy collisions/ A. Anselm and M. Ryskin// Phys. Lett B 226, 482 (1991).

10. Blaizot, J.P. Soft-pion emission in high-energy heavy-ion collisions/ J.-P. Blaizot and Krzwitski// Phys. Rev. D 46, 246 (1992).

11. Bjorken, J.D. A full-acceptance detector for SSC physics at low and intermediate mass scales: an expression of interest to the SSC/ J.D. Bjorken// Intern. J. Mod. Phys. A 7, 4189 (1992).

12. Mishustin, I.N. Multipion droplets/ I.N. Mishustin and W. Greiner// J. Phys. G: Nucl. Part. Phys. 19, L101 (1993).

13. Son, D.T. QCD at Finite Isospin Density/ D.T. Son and M.A. Stephanov// Phys. Rev. Lett. 86, 592 (2001); [arXiv:hep-ph/0005225]; Phys. Atom. Nucl. 64, 834 (2001).

14. Kogut, J. QCD at small non-zero quark chemical potentials/ J. Kogut and D. Toublan// Phys. Rev. D 64, 034007 (2001); [arXiv:hep-ph/0103271].

15. Toublan, D. Isospin Chemical Potential and the QCD Phase Diagram at Nonzero Temperature and Baryon Chemical Potential/ D. Toublan and J. Kogut// Phys. Lett. B 564, 212 
(2001); [arXiv:hep-ph/0301183].

16. Mammarella, A. Intriguing aspects of meson condensation/ A. Mammarella and M. Mannarelli// Phys. Rev. D 92, 085025 (2015); [1507.02934 [hep-ph]], 2015.

17. Carignano, S. Scrutinizing the pion condensed phase/ S. Carignano, L. Lepori, A. Mammarella, M. Mannarelli, G. Pagliaroli// Eur. Phys. J. A 53, 35 (2017); [arXiv:1610.06097 [hep-ph]].

18. Brandt, B.B. QCD phase diagram with isospin chemical potential/ B.B. Brandt, G. Endrodi// PoS LATTICE2016 039 (2016); [arXiv:1611.06758 [hep-lat]].

19. Brandt, B.B. QCD at finite isospin chemical potential/ B.B. Brandt, G. Endrodi, S. Schmalzbauer// arXiv:1709.10487 [hep-lat].

20. Anchishkin, D. Phase Transition in Interacting Boson System at Finite Temperatures/ D. Anchishkin, I. Mishustin, and H. Stoecker// J. Phys. G. 46, No.3, 035002 (2019); arXiv:1806.10857 [nucl-th].

21. Satarov, L.M. Equation of state of hadron resonance gas and the phase diagram of strongly interacting matter/ L.M. Satarov, M.N. Dmitriev, I.N. Mishustin // Phys. Atom. Nucl. 72, 1390-1415 (2009).

22. Vovchenko, V. Multicomponent van der Waals equation of state: Applications in nuclear and hadronic physics/ V. Vovchenko, A. Motornenko, P. Alba, M.I. Gorenstein, L.M. Satarov and H. Stoecker // Phys. Rev. C 96, 045202 (2017).

23. Anchishkin, D. Mean-field approach in the multi-component gas of interacting particles applied to relativistic heavy-ion collisions/ D. Anchishkin, V. Vovchenko// J. Phys. G: Nucl. Part. Phys. 42, 105102: 1-27 (2015); arXiv:1411.1444 [nucl-th].

24. Anchishkin, D.V. Particle finite-size effects as a mean-field approximation/ D.V. Anchishkin// Sov. Phys. JETP 75, 195 (1992) [Zh. Eksp. Teor. Fiz. 102, 369 (1992)].

25. Anchishkin, D. Generalization of mean-field models to account for effects of excludedvolume / D. Anchishkin, E. Suhonen // Nucl. Phys. A 586, 734-754 (1995).

26. Anchishkin, D. Bose-Einstein Condensation in Interacting Boson Particle-Antiparticle System/ D. Anchishkin, V. Gnatovskyy, D. Zhuravel, and V. Karpenko // To be published.

27. Poberezhnyuk, R.V. Limiting temperature of pion gas with the van der Waals equation of state/ R.V. Poberezhnyuk, V. Yu. Vovchenko, D.V. Anchishkin, M.I. Gorenstein // J. Phys. G: Nucl. Part. Phys. 43, 095105 (2016); arXiv:1508.04585 [nucl-th].

28. Anchishkin, D. Finite-Temperature Bose-Einstein Condensation in Interacting Boson System/ D. Anchishkin, I. Mishustin, O. Stashko, D. Zhuravel, H. Stoecker // Ukranian J. Phys. 64, No. 12, 1110-1116 (2019).

29. Shuryak, E.V. Collective interaction of mesons in hot hadronic matter/E.V. Shuryak// Nucl. Phys. A 533, 761 (1991).

30. Theis, J. Phase transition of the nucleon-antinucleon plasma in a relativistic mean-field theory / J. Theis, G. Graebner, G. Buchwald, J. Maruhn, W. Greiner, H. Stoecker, J. Polonyi // Phys. Rev. D 28, 2286 (1983).

31. Hansen, J.P. Theory of Simple Liquids / J.P. Hansen, I.R. McDonald // Academic Press, 2006.

32. Stashko, O.S. Thermodynamic properties of interacting bosons with zero chemical potential/ O.S. Stashko, D.V. Anchishkin, O.V. Savchuk, and M.I. Gorenstein // J. Phys. G: Nucl. Part. Phys. (2020) doi: 10.1088/1361-6471/abd5a5; arXiv: 2007.06321 [hep-ph].

33. Kataja, M. Nonzero chemical potential and the shape of the $p_{T}$ distribution of hadrons in heavy-ion collisions/ M. Kataja and P.V. Ruuskanen // Phys. Lett. B 243, 181 (1990). 
34. London, F. The $\lambda$-Phenomenon of Liquid Helium and the Bose-Einstein Degeneracy/ F. London // Nature 141, 643 (1938).

35. Ehrenfest, P. Phasenumwandlungen im ueblichen und erweiterten Sinn, classifiziert nach dem entsprechenden Singularitaeten des thermodynamischen Potentiales / P. Ehrenfest // Communications from the Physical Laboratory of the University of Leiden, Supplement No. 75b (1933).

36. Jaeger, Gregg The Ehrenfest Classification of Phase Transitions: Introduction and Evolution/ Gregg Jaeger// Arch. Hist. Exact Sci. 53, 51 (1998).

37. Mishustin, I. Condensation of interacting scalar bosons at finite temperatures/ I. Mishustin, D. Anchishkin, L. Satarov, O. Stashko, H. Stoecker // Phys. Rev. C 100, 022201(R) (2019).

38. Walecka, J.D. A theory of highly condensed matter/ J.D. Walecka// Ann. Phys. (N.Y.) 83, 491 (1974).

39. Serot, B. D. The Relativistic Nuclear Many-Body Problem / B.D. Serot and J.D. Walecka // Adv. Nucl. Phys. 16 (1986).

40. Waldhauser, B.M. Nuclear equation of state from the nonlinear relativistic mean field theory / B.M. Waldhauser, J.A. Maruhn, H. Stöcker, W. Greiner // Phys. Rev. C 38, 1003 (1988). 